\title{
MS26-01 | Some Recent Advances in the Surface Science of Complex Metallic
}

\section{AlLoys}

McGrath, Ronan (University of Liverpool, Oxford Street, Liverpool, GBR)

Complex metallic alloys present unique challenges and opportunities in surface science, both for preparation of clean surfaces and for epitaxial studies.

Studies of three $A g-\operatorname{In}-\mathrm{RE}(100)(\mathrm{RE}=\mathrm{Yb}, \mathrm{Gd}, \mathrm{Tb})$ approximants illustrate a variety of surface behaviours when prepared in ultra-high-vacuum conditions [1] from flat surfaces to multiple faceting; I will discuss the origin these behaviours in terms of surface atomic bonding.

In recent work, we demonstrated that $C_{60}$ molecules adsorbed on this surface at room temperature form a Fibonacci square grid - the first physical manifestation of a prediction made in 2002 by Lifshitz [2]. In this case the $\mathrm{C}_{60}$ molecules bond preferentially to $\mathrm{Mn}$ atoms in the surface layer, which are arranged in the Fibonacci square grid geometry [2].

In a further study, the adsorption of $\mathrm{Pb}$ atoms on the 3-fold surface of the icosahedral Ag-In-Yb quasicrystal proceeds in an unusual way; instead of layer-by-layer growth as was observed on the five-fold surface [3], the atoms form quasicrystalline $\mathrm{Pb}$ nanoclusters by mimicking the structure of the underlying substrate. I will discuss whether this is long-sought evidence for "cluster stability" in quasicrystals.

[1] S. Hars, H. R. Sharma, J. A. Smerdon, T. P. Yadav, A. Al-Mahboob, J. Ledieu, V. Fournée, R. Tamura, and R. McGrath, Phys. Rev. B 93 (2016) 205428

[2] S. Coates, J.A. Smerdon, R. McGrath \& H. R. Sharma, Nature Communications 9 (2018) 3435.

[3] S. Coates, S. Thorn, R. McGrath and H.R. Sharma, in preparation. 